\title{
INTEGRAL monitoring of the X-ray burster XTE J1739-285
}

\section{Celia Sánchez-Fernández ${ }^{* \dagger}$}

ISOC, ESA/ESAC, Urbanización Villafranca del Castillo, PO Box 5072728080 Madrid, Spain

E-mail: Celia.Sanchez@sciops.esa.int

\section{Erik Kuulkers}

ISOC, ESA/ESAC, Urbanización Villafranca del Castillo, PO Box 5072728080 Madrid, Spain

E-mail: Erik.Kuulkersesciops.esa.int

\section{Jérôme Chenevez, Søren Brandt}

DTU Space - National Space Institute, Juliane Maries Vej, 302100 Copenhagen, Denmark

E-mail: jerome@space.dtu.dk, sb@space.dtu.dk

\section{Antonella Tarana, Angela Bazzano}

IASF-Rome/INAF

E-mail: antonella.tarana@iasf-roma.inaf.it,

angela.bazzanodiasf-roma.inaf.it

XTE J1739-285 is a recurrent X-ray transient first discovered by INTEGRAL as an X-ray burster. We have carried out a systematic search for X-ray bursts at various levels of accretion rate onto the Neutron Star surface during the source outbursts in 2005 and 2006. A total of 25 X-ray bursts were found as a result of this analysis. Their main properties are studied here, and are related to the overall source state by the time of burst occurrence.

7th INTEGRAL Workshop

September 8-112008

Copenhagen, Denmark

*Speaker.

${ }^{\dagger}$ On behalf of the Galactic Bulge monitoring group 


\section{Introduction}

XTE J1739-285 is a Low Mass X-ray Binary (LMXRB) discovered during Rossi X-ray Timing Explorer (RXTE) Proportional Counter Array (PCA) scans of the Galactic bulge[1]. The source is a recurrent transient and several outbursts have been detected after its discovery. However, little was known about the nature of the compact object, until it was first detected as an X-ray burster by the Joint European Monitor for X-rays (JEM-X) onboard INTEGRAL [2]. Later, coherent sub-millisecond oscillations $(v=1122 \pm 0.3 \mathrm{~Hz})$ were detected during the tail of one bright X-ray burst detected by RXTE [3]. If related to the Neutron Star spin, this frequency suggests that XTE J1739-285 might harbour the fastest rotating Neutron Star yet known.

The source is located in the Galactic Center Field, frequently visited by INTEGRAL by means of Guest Observer (GO) and Core Programme (CP) observations. We have compiled the available INTEGRAL observations during 2005-2006, when the transient source experienced two outbursts, and carried out a systematic search for X-ray bursts. The results of this analysis are presented here, and discussed in relation with the overall source properties. A more detailed analysis of these data is presented in [4].

\section{Observations and data reduction}

We have analyzed INTEGRAL data from all the pointings when XTE J1739-285 was located within the Fully Coded Field of View of JEM-X ( $4.8 \times 4.8$ degrees $)$, in 4 visibility periods. We have used private data from the INTEGRAL Galactic Bulge monitoring programe [5], (revs. 287-307; 347-370; 406-429, 469-422) Core Programme observations of the Galactic Center (the so-called Galactic Center Deep Exposure, GCDE; revs. 353-364 and 409) and have complemented these observations with the available public exposures for which XTE J1739-285 is contained in the JEM-X field of view, this mainly corresponds to data in revs. 409-428. In this work we only consider data from JEM-X and IBIS/ISGRI. We do not consider the data from IBIS/PICsIT, SPI or OMC instruments because either the angular resolution is limited (SPI: $2.5^{\circ}$ ) and therefore not good enough to disentagle XTE J1739-285 from the vicine sources in the crowded Galactic Center region, or the source is not hard enough to be detected by IBIS/PICsIT, (which operates at $>175$ $\mathrm{keV}$ ), or, being located in the GC region, the source is highly obscured, therefore not detectable by the OMC.

JEM-X and IBIS/ISGRI data have been analyzed through the imaging step to the light-curve step using the latest version of the Offline Science Analysis software (OSA v7.0, September 2007) released by the INTEGRAL Science Data Centre (ISDC, [6]). Analysis has been run using the default parameters. Light curves have been extracted using two different time resolutions: a science window time resolution, to derive the long term properties of the system, and 5-second time resolution to search for X-ray bursts and characterise their properties. The spectral analysis was carried out with XSPEC v 12.4 [7].

We have developed IDL-based burst searching routines to systematically search for X-ray bursts in the source (JEM-X) light curves. These routines have been run on the 5 -second binned 3.5-15 keV Energy band. This Energy range has been selected after verifying that it contains $95 \%$ of the burst energy output for this source. 


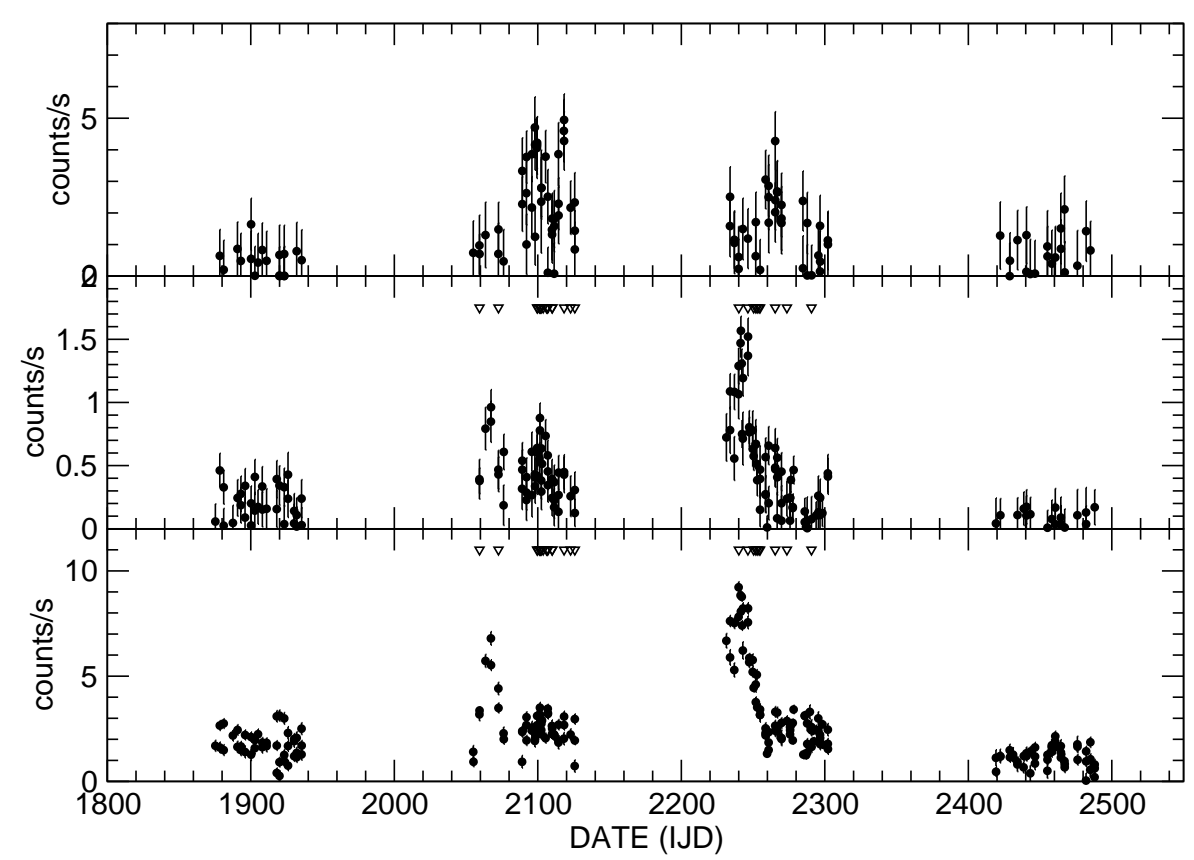

Figure 1: X-ray light curve of XTE J1739-285 as observed by INTEGRAL in three energy bands: 3.5$10 \mathrm{keV}$ (JEM-X, bottom panel), 10-20 keV (JEM-X, med. panel) and 18-40, keV (IBIS/ISGRI) top panel. Data points are one day averages. The times of detection of X-ray bursts are shown by arrow heads in the middle and lower panels.

\section{Results}

\subsection{Persistent emission: light curves}

The light curve of XTE J7139-285 as observed by IBIS/ISGRI and JEM-X during the four visibility periods analyzed in this work is shown in figure 1 . In order to characterise the spectral evolution of XTE J1739-285 in this epoch, we have extracted the source lights curves in three energy bands: 3.5-10 keV (JEM-X; lower panel), 10-20 keV (JEM-X; mid-panel) and 18-40 keV (IBIS/ISGRI; upper panel). During the first and fourth visibility periods, XTE J1739-285 remained in quiescence, whereas in the second and third visibility periods it underwent two outbursts in Xrays. These outbursts had durations of the order of 30-40 days in soft X-rays and reached peak fluxes $\sim 10^{-9} \mathrm{erg} \mathrm{cm}^{-2} \mathrm{~s}^{-1}$. The recurrence time between the outbursts was 170 days (from onset to onset). The spectral evolution was very similar during both outbursts: the outbursts started first in soft X-rays (below $20 \mathrm{keV}$ ), while no substantial emission was detected above $20 \mathrm{keV}$. The soft emission peaked to a maximum in 10 days and then decayed linearly during the subsequent 10 20 days. The decay in soft X-rays was followed by an increase in the hard X-ray emission. The spectral evolution of the source during outburst is discussed in further detail in [4]. 

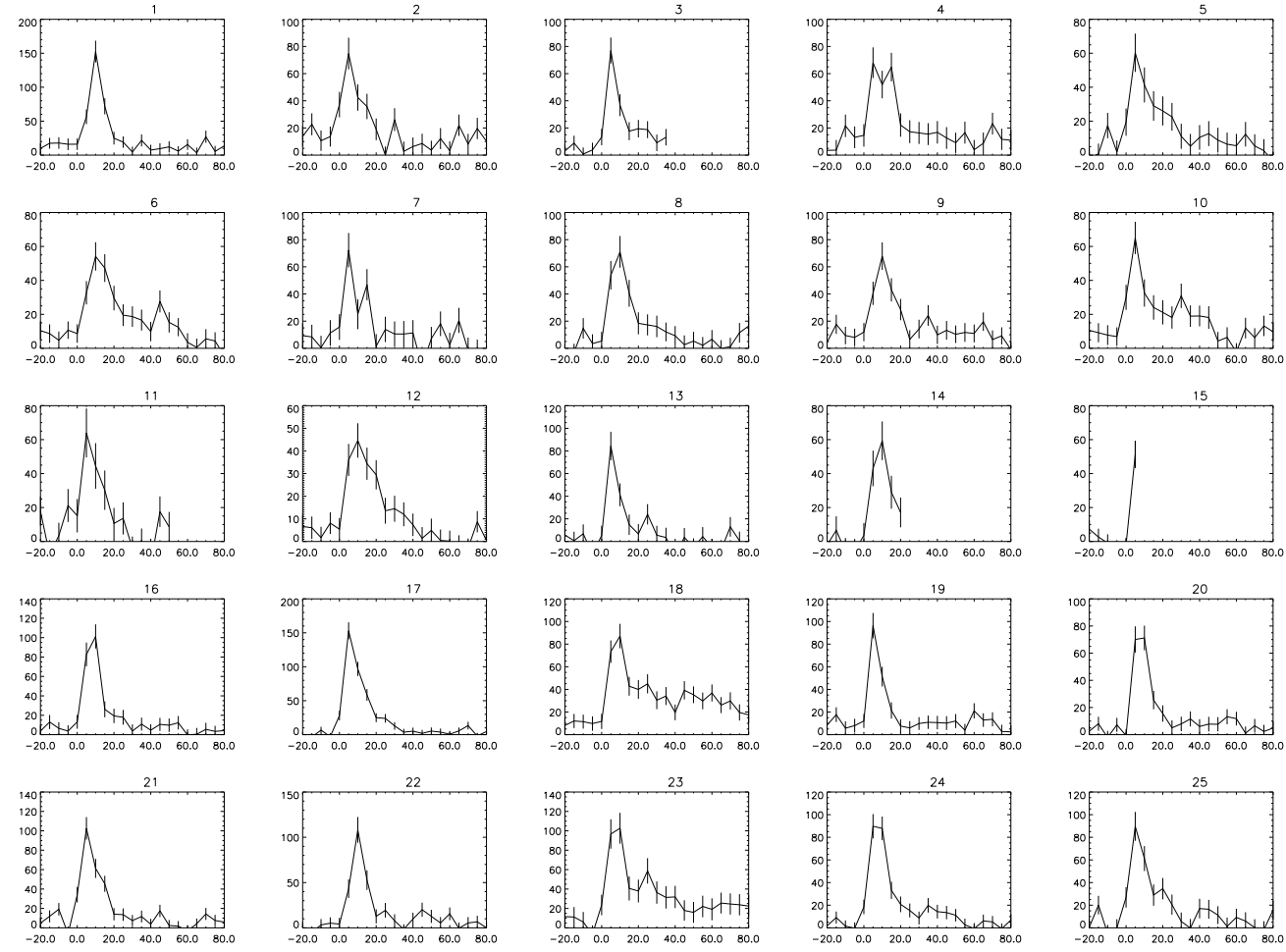

Figure 2: The 25 Type I X-ray bursts of XTE J1739-285 detected by JEM-X onboard INTEGRAL during 2005-2006. The light curves are shown in the 3.5-15.0, keV band, which contains $\sim 95 \%$ of the burst energy. Time bin is $5 \mathrm{sec}$. The horizontal axis units are seconds since the beginning of the burst. The vertical axis are $\mathrm{cts} \mathrm{s}^{-1}$. Bursts are numbered 1-25 to allow reference to the the burst parameters provided in Table 1.

\subsection{X-ray bursts: time scales and main parameters}

We detected 25 thermonuclear X-ray bursts from XTE J1739-285 during 2005-2006. For reference, their times of occurrence are shown in Figure 1. Note that X-ray bursts were only detected during outburst, no X-ray burst was detected during periods of quiescence. The individual burst profiles in the $3.5-15.0 \mathrm{keV}$ band are shown in Figure 2, and their main properties in that energy band are summarized in table 1 .

For each burst we have measured the peak count rate, persistent emission by the time of burst detection and burst duration, computed as the time elapsed since the rising count rate reached a value equal or larger than $5 \%$ of the burst peak, until it decayed to $5 \%$ of the peak count rate. The burst e-folding time was derived by fitting the decay light curve with an exponential function $\left(\mathrm{e}^{-\mathrm{t} / \tau_{\exp }}\right)$. We have have also computed the integrated burst count rate (or fluency) as the integral of the exponential decay function between the time of maximum and infinity, i.e., as $\mathrm{C}_{\text {peak }} \times \tau_{\text {exp }}$.

With the exception of the X-ray burst \# 18, which displayed a long tail in soft X-rays, all the $\mathrm{X}$-ray bursts we observed were short, with rise times $\sim 2$ seconds, and average e-folding decay times of 8.67 seconds. The X-ray burst \# 18 which was detected during the peak of the secound outburst, displayed a long tail in soft X-rays with a duration $>150$ seconds. Unfortunately, this burst was detected close to the end of a science window and we missed the tail of the decay to quiescence. Its main properties are discussed in further detail in [4]. 


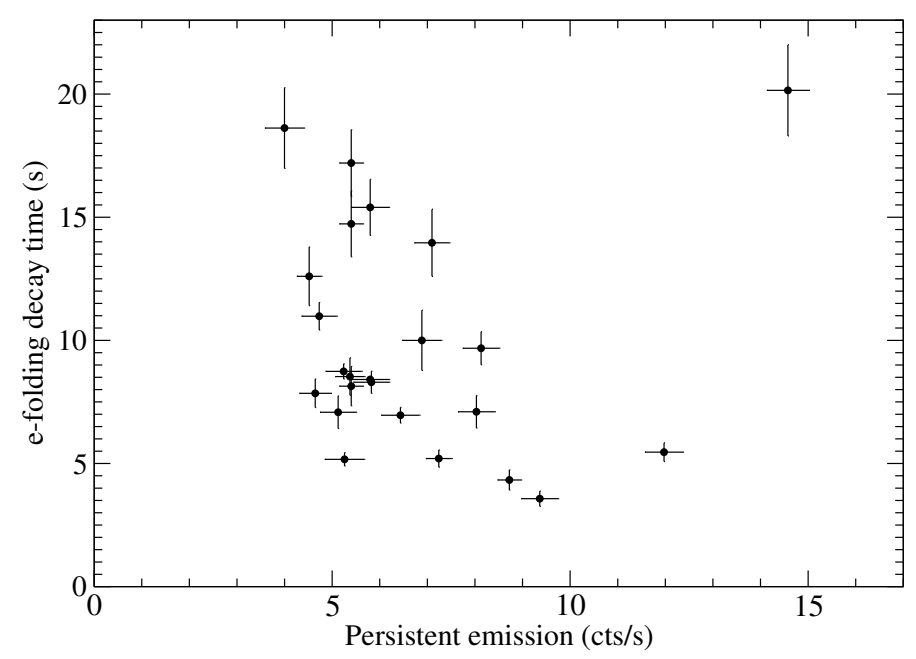

Figure 3: Burst e-folding decay time $\left(\tau_{\text {exp }}\right)$ as a function of persistent emission in the $3.5-15.0 \mathrm{keV}$ band. The X-ray bursts detected at higher levels of persistent emission tend to decay faster, than those bursts detected during periods of lower accretion rates, which display slower decays e-folding decay time. The exception to this trend is the X-ray burst \# 18, which corresponds to the point at the upper right side of the plot.

We explore in Figure 3 and 4 relations between some of the burst parameters. Figure 3 shows the burst e-folding decay time as a function of the source persistent emission by the time of burst detection. The plot shows that those bursts detected during lower levels of persistent emission, decay slower than those detected during periods of higher accretion rate, which tend to decay faster. The exception to this trend is the X-ray burst \# 18, which had a long tail in X-rays and was detected close to the maximum of the second outburst. The point in the upper right corner of the plot corresponds to the X-ray burst number \# 18. Excluding it from the calculation, we measure a Spearman's (rho) rank correlation of -0.25 between the e-folding decay time $\left(\tau_{\text {exp }}\right)$ and the level of persistent emission at the time of burst detection. We present in Figure 4 the relation between the burst e-folding decay time $\left(\tau_{\text {exp }}\right)$, and the burst peak count rate. The plot shows that X-ray burst with a higher peak count rate decay faster than those for which we measure a lower peak count rate. The X-ray burst \# 18 also follows the trend.

\section{Summary of results and discussion}

We have monitored the transient X-ray burster XTE J1739-285 over various levels of accretion during the period 2005-2006, when the source experienced 2 outbursts in X-rays. During this monitoring, we have detected 25 Type I X-ray bursts from the system, all of them occurring when the source was in outburst.

We have measured the main parameters of the detected X-ray bursts and have explored the relations between then. Our study reveals an anticorrelation between burst peak count rate and burst duration: more intense X-ray bursts tend to decay faster than weaker ones. We have also found an anticorrelation between the decay e-folding time $\left(\tau_{\text {exp }}\right)$ and the level of persistent emission by the 


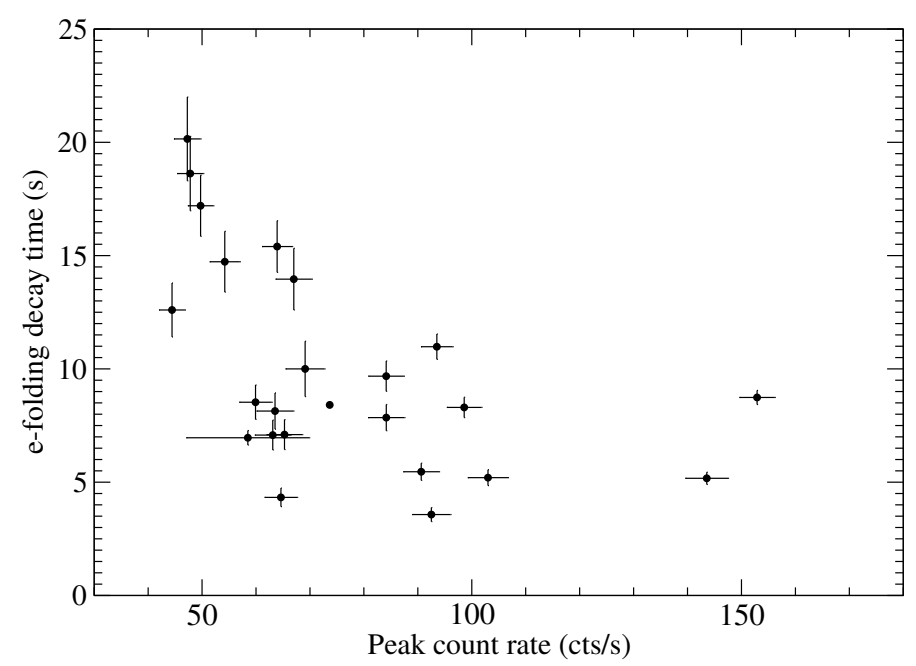

Figure 4: Burst e-folding decay time as a function of burst peak count rate in the $3.5-15 \mathrm{keV}$ band. The $\mathrm{X}$-ray bursts with higher peak count rate tend to decay faster than those displaying lower peak count rates.

time of burst detection: those X-ray bursts detected at lower levels of persistent emission decay more slowly than those detected at higher levels of persistent emission.

These correlations suggest a variation in the composition of the ignited shell as a function of accretion rate onto the neutron star. According to burst theory, the nuclear ashes and burst properties depend on the amount of $\mathrm{H}$ vs $\mathrm{He}$ present at the time of ignition of an X-ray burst [8]. He burning proceeds rapidly via strong interactions, while $\mathrm{H}$ burning is relatively slow, since $\beta$ decays are required to convert protons to neutrons. Therefore, the properties of the bursts provide a clue to the composition of the burning layer, which for solar metalicities is actually driven by the accretion rate in the following way[8, 9]: at the lowest accretion rates $\left(10^{-14} \lesssim \dot{M}<2 \times 10^{-10}\right.$ $\mathrm{M}_{\odot} \mathrm{yr}^{-1}$ ) bursts are triggered by thermally unstable $\mathrm{H}$ burning, in turn triggering $\mathrm{He}$ burning. At intermediate accretion rates $\left(2 \times 10^{-10} \lesssim \dot{\mathrm{M}}<2 \times 10^{-9} \mathrm{M}_{\odot} \mathrm{yr}^{-1}\right) \mathrm{H}$ burns stably into He between bursts, leading to a growing pure He layer at the base of the accreted material. The fuel layer heats steadily until $\mathrm{He}$ ignition occurs and the He burns via the triple- $\alpha$ process. At these temperatures and pressures, He burning is extremely unstable, and a rapid and intense He burst follows. At yet higher accretion rates $\left(10^{-9} \lesssim \dot{M}<2.6 \times 10^{-8} \mathrm{M}_{\odot} \mathrm{yr}^{-1}\right) \mathrm{H}$ is accreted faster that in can be consumed by steady burning (limited by the $\beta$-decays in the CNO cycle) so that He ignites unstably in a H-rich environment, this can significantly delay the cooling of the burning layer, giving longer burst tails.

In our sample of X-ray bursts, the bursts tend to decay faster as the persistent emission increases. This suggests that the fraction of burning $\mathrm{H}$, which causes longer tails, is lower as accretion rate increases, likely due to steady burning of $\mathrm{H}$ between bursts, This increases the fraction of $\mathrm{He}$, which produces shorter, more intense bursts, in agreement with the inverse relation we find between burst peak count rate, and e-folding decay time: shorter bursts in our sample present higher peak count rates. More detailed discussions are provided in [4]. 
Table 1: Properties of the $25 \mathrm{X}$-ray bursts analyzed in this work. Values refer to the $3.5-15.0 \mathrm{keV}$ energy band, and have been calculated on the 5-sec binned burst light curves. Bursts \# 3, 15, 17 and 18 were detected close to the end of their respective science windows, therefore burst durations are provided as lower limits. Burst amplitude is the (persistent emission subtracted) burst peak count rate.Fluency has been computed from the product $\mathrm{C}_{\text {peak }} \times \tau_{\text {exp }}$, as explained in the text. For each $\mathrm{X}$-ray burst we provide the recurrence time, computed as the time elapsed since the onset of the previous X-ray burst. Note, however, that for X-ray bursts \# 1, 18, detected at the beginning of a visibility period, this information cannot be provided.

\begin{tabular}{|c|c|c|c|c|c|c|c|c|}
\hline \# & $\begin{array}{l}\text { Start time } \\
\text { (IJD) }\end{array}$ & $\begin{array}{l}\text { Off-axis } \\
\text { (deg) }\end{array}$ & $\begin{array}{l}\text { Duration } \\
(\mathrm{sec})\end{array}$ & $\begin{array}{l}\text { Ampl } \\
\text { (cts/s) }\end{array}$ & $\begin{array}{r}\tau_{\exp } \\
\left(\sec ^{-1}\right)\end{array}$ & $\begin{array}{r}\text { pers emission } \\
(\mathrm{cts} / \mathrm{s})\end{array}$ & $\begin{array}{l}\text { Fluency } \\
\text { (counts) }\end{array}$ & $\begin{array}{r}\text { Recurrence } \\
(\mathrm{min})\end{array}$ \\
\hline 1 & 2059.37395 & 2.8 & 20 & $143 \pm 4$ & $5.2 \pm 0.3$ & $5.3 \pm 0.4$ & $730 \pm 60$ & - \\
\hline 2 & 2072.57230 & 2.7 & 20 & $66 \pm 3$ & $7.1 \pm 0.7$ & $8.0 \pm 0.4$ & $460 \pm 70$ & 19005.5 \\
\hline 3 & 2099.49486 & 1.4 & $>30$ & $73 \pm 0$ & $8.4 \pm 0.1$ & $5.8 \pm 0.3$ & $619 \pm 8$ & 38768.6 \\
\hline 4 & 2099.89766 & 1.7 & 55 & $62 \pm 3$ & $15.4 \pm 1.1$ & $5.8 \pm 0.3$ & $992 \pm 100$ & 580.078 \\
\hline 5 & 2101.22383 & 3.1 & 30 & $54 \pm 3$ & $14.7 \pm 1.3$ & $5.4 \pm 0.2$ & $822 \pm 100$ & 1909.69 \\
\hline 6 & 2101.51561 & 0.4 & 50 & $50 \pm 2$ & $17.2 \pm 1.3$ & $5.4 \pm 0.2$ & $836 \pm 100$ & 420.117 \\
\hline 7 & 2101.78005 & 2.5 & 15 & $70 \pm 3$ & $8.1 \pm 0.8$ & $5.4 \pm 0.2$ & $508 \pm 80$ & 380.742 \\
\hline 8 & 2102.44812 & 2.7 & 35 & $64 \pm 3$ & $7.1 \pm 0.7$ & $5.1 \pm 0.4$ & $440 \pm 60$ & 961.875 \\
\hline 9 & 2103.74367 & 1.7 & 15 & $61 \pm 3$ & $8.5 \pm 0.8$ & $5.4 \pm 0.3$ & $500 \pm 70$ & 1865.74 \\
\hline 10 & 2106.08578 & 1.7 & 45 & $58 \pm 2$ & $18.6 \pm 1.6$ & $4.0 \pm 0.4$ & $878 \pm 100$ & 3372.54 \\
\hline 11 & 2106.58079 & 4.0 & 25 & $57 \pm 4$ & $10.0 \pm 1.2$ & $6.9 \pm 0.4$ & $691 \pm 120$ & 712.969 \\
\hline 12 & 2107.01402 & 0.8 & 35 & $40 \pm 2$ & $12.6 \pm 1.2$ & $4.5 \pm 0.2$ & $571 \pm 80$ & 623.672 \\
\hline 13 & 2109.99659 & 2.0 & 35 & $83 \pm 3$ & $7.9 \pm 0.6$ & $4.6 \pm 0.3$ & $672 \pm 80$ & 4295.04 \\
\hline 14 & 2110.02616 & 2.0 & $>20$ & $58 \pm 1$ & $7.0 \pm 0.3$ & $6.4 \pm 0.4$ & $385 \pm 90$ & 42.5391 \\
\hline 15 & 2118.29871 & 2.5 & $>10$ & $70 \pm 1$ & & $5.7 \pm 0.4$ & & 11912.7 \\
\hline 16 & 2122.82200 & 2.6 & 20 & $94 \pm 4$ & $3.6 \pm 0.3$ & $9.3 \pm 0.4$ & $331 \pm 40$ & 6513.40 \\
\hline 17 & 2125.75040 & 1.4 & 30 & $150 \pm 3$ & $8.7 \pm 0.3$ & $5.2 \pm 0.4$ & $1353 \pm 80$ & 4216.99 \\
\hline 18 & 2240.06711 & 1.9 & $>150$ & $76 . \pm 2$ & $20.1 \pm 1.9$ & $14.6 \pm 0.4$ & $952 \pm 140$ & - . \\
\hline 19 & 2246.41857 & 1.5 & 15 & $87 \pm 3$ & $5.5 \pm 0.4$ & $12.0 \pm 0.4$ & $500 \pm 50$ & 9145.90 \\
\hline 20 & 2250.40831 & 0.6 & 15 & $68 \pm 3$ & $4.3 \pm 0.4$ & $8.7 \pm 0.2$ & $280 \pm 40$ & 5745.23 \\
\hline 21 & 2251.87847 & 1.5 & 35 & $97 \pm 3$ & $8.3 \pm 0.5$ & $5.8 \pm 0.4$ & $812 \pm 70$ & 2117.11 \\
\hline 22 & 2252.86627 & 3.0 & 25 & $105 \pm 4$ & $5.2 \pm 0.4$ & $7.2 \pm 0.3$ & $531 \pm 50$ & 1422.42 \\
\hline 23 & 2254.17074 & 3.0 & 40 & $86 \pm 3$ & $14.9 \pm 1.4$ & $7.1 \pm 0.4$ & $935 \pm 140$ & 1878.40 \\
\hline 24 & 2254.85736 & 1.5 & 50 & $86 \pm 3$ & $11.9 \pm 0.6$ & $4.7 \pm 0.4$ & $1046 \pm 90$ & 988.945 \\
\hline 25 & 2273.59229 & 2.1 & 25 & $82 \pm 3$ & $9.7 \pm 0.7$ & $8.1 \pm 0.4$ & $828 \pm 90$ & 26978.2 \\
\hline
\end{tabular}

\section{References}

[1] Markwardt, C. B.; Marshall, F. E.; Swank, J. H.; Wei, Cui (1999) IAU Circ. 7300

[2] Brandt, S. et al. (2005) The Astronomer's Telegram, \#622

[3] Kaaret, P. et al. (2007), ApJ, 657, 97

[4] Sánchez-Fernández et al., in preparation

[5] Kuulkers, E., et al., (2007) A\&A, 466, 595

[6] Courvoisier, T. J.-L. et al., (2003) A\&A, 411, 53

[7] Arnaud, K. A. Astronomical Data Analysis Software and Systems V, A.S.P. Conference Series, Vol. 101, (1996)

[8] Fujimoto, M. Y.; Hanawa, T.; Miyaji, S., (1981) ApJ, 247, 267

[9] Cumming, A., Bildsten, L., (2000) ApJ, 544, 453 